\title{
Quality of Life in Stroke Patients Using SSQoL Scale and Barthel Index
}

\author{
Mohanraj Rathinavelu Mudaliarr,2, Samhitha Reddy Yiragamreddy', Priyanka Pichala Tejashwani', \\ Sowjanya Umapathi ${ }^{*}$, Nagamani Sake ${ }^{1}$, Sreedhar Sharma ${ }^{3}$
}

${ }^{1}$ Division of Pharmacy Practice, Raghavendra Institute of Pharmaceutical Education and Research (RIPER), Anantapuramu, Andhra Pradesh, INDIA.

${ }^{2}$ Drug Information Pharmacist, Poison and Drug Information Center, Rural Development Trust (RDT) Hospital Bathalapalli, Anantapuramu, Andhra Pradesh, INDIA.

${ }^{3}$ Department of Nephrology, Government Medical College and Hospital, Anantapuramu, Andhra Pradesh, INDIA.

\begin{abstract}
Introduction: Stroke is a major catastrophic event majorly defining morbidity and mortality in patients. The assessment of quality of life after stroke is becoming common with the recognition that evaluation of treatment should include quality as well as quantity of survival. Materials and Methods: The current prospective, observational study of six months duration was performed to assess the quality of life in stroke inpatients of a tertiary care teaching hospital in south India using SSOoL scale and Barthel Index. The study participants were interviewed based on 12 domains and 49 items of SSOoL scale and 10 questions from Barthel index. Results: Out of 48 stroke inpatients, $62.5 \%$ were male and $37.5 \%$ female, furthermore there was equal distribution of patients with respect to gender within age groups of 56-60 and 61-65 years. The changes in quality of life observed in all stroke inpatients using SSOoL scale and Barthel Index with respect to associated complications was found to be higher in hypertension populations, patients with tobacco and pan chewing habit was observed as primary risk factor for stroke attacks, and patients with $1^{\text {st }}$ episode without accident proved more risk for stroke attack. Conclusions: In conclusion, improved methods to measure the quality of life in stroke are required. Quality of life measures must be valid, reliable, responsive and comprehensive; and also with the involvement of patients at every stage of measure. Performing an exemplary and extensive research studies on stroke and its quality of life could bring better health outcome in individual patients.
\end{abstract}

Key words: Cerebro vascular accidents, Barthel Index, Inpatients, Quality of life, SSQoL.

\section{INTRODUCTION}

The World Health Organization (WHO) definition of stroke is: "rapidly developing clinical signs of focal (or global) disturbance of cerebral function, with symptoms lasting $24 \mathrm{~h}$ or longer or leading to death, with no apparent cause other than of vascular origin". ${ }^{1}$ The 1990 Global Burden of Disease (GBD) study provided the first global estimate on the burden of 135 diseases, and cerebrovascular diseases ranked as the second leading cause of death after ischemic heart disease. ${ }^{2}$

According to the India stroke factsheet updated in 2012, the estimated age-adjusted prevalence rate for stroke ranges between $84 / 100,000$ and 262/100,000 in rural and between 334/100,000 and 424/100,000 in urban areas. $^{3}$

After coronary heart disease (CHD) and cancer of all types, stroke is the third commonest cause of death worldwide. However unlike the Caucasians, Asians have a lower rate of $\mathrm{CHD}$ and a higher prevalence of stroke. ${ }^{4}$ Studies from Asian countries indicate that the proportion of ICH is higher than in Caucasians with approximately $20 \%$ to $30 \%$ being hemorrhagic. ${ }^{5,6,7,8,9}$ Higher numbers are criticized for being due to lack of CT scanning and for not complying with standard criteria for stroke identification. ${ }^{6,7} \mathrm{~A}$ recent review on stroke epidemiology data in
DOI: 10.5530/ijopp.11.1.8

Address for correspondence: Dr. Mohanraj M. Rathinavelu. Pharm.D,

Associate Professor and Internship Coordinator, Division of Pharmacy Practice, Raghavendra Institute of Pharmaceutical Education and Research (RIPER), Anantapuramu, Andhra Pradesh, INDIA.

Phone no: +91 8121934940 Email Id: moley4u@rediffmail. com

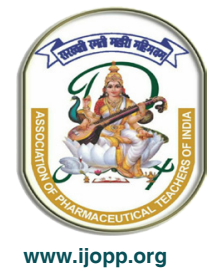


Hong Kong, Taiwan, South Korea, Singapore, Malaysia, Thailand, Philippines and Indonesia, reported that the proportion of ischemic and hemorrhagic strokes varied from $17 \%$ to $33 \%{ }^{8}$

Although the stroke mortality rate has been declining, ${ }^{10}$ the estimated prevalence rate of stroke-related disability is about 331 per $100,000 .{ }^{11}$ Stroke disability and morbidity cause reduced quality of life (QoL) among stroke survivors. ${ }^{12}$ The greater the disability, the lower the QoL is. ${ }^{13}$

Quality of Life (QoL) assessment has been an important part of the evaluation of stroke patients and their treatment for more than 30 years. QoL is difficult to define and no universal definition of this term exists. However, there is a general agreement that QoL is a multi-dimensional construct that consists of at least three broad domains: physical, mental and social. Researchers and physicians have often used the health-related quality of life concept in the field of medicine, which specifically focuses on the impact of an illness and/or the treatment on the patients' perception, of their status of health, and, on subjective well-being or satisfaction with life. ${ }^{14}$ The impact of stroke on health related quality of life may be disastrous; stroke can affect multiple domains of life. To assess these consequences several instruments have been developed. Most of them are questionnaires based on a patient's subjective self-report or selfevaluation. Some of these tools provide information about perceived health status, for example: physical and mental functions, ability to perform everyday activities/ roles or the limitation in performing these activities/roles. The other scales capture an assessment of well-being or positive/negative evaluation of particular life domains or satisfaction with life (or specific life domains). There are also questionnaires which produce both information about perceived health status and subjective evaluation. ${ }^{15}$

Quality of life measures should be used in stroke patients because they are multidimensional instruments which comprise functional, physical, cognitive, psychological and social elements. Additionally they are patient centered which fairly accurately represent patients perspective of health and a given treatment. Quality of life instruments also measure quality as well as quantity of the further life. ${ }^{16}$ For a rationale individual management programme, outcome measurement is highly important in stroke. The care of a stroke patient requires objective measurements of outcome, which are critical to assessment and evaluations of treatment regimens. In stroke, traditional outcome assessment focuses on prevention of deaths, alleviation of symptoms and restoration of function. In this context, the current prospective, observational study was performed to assess the quality of life (QoL) using Stroke specific QoL scale ${ }^{16}$ and Barthel index ${ }^{17}$ in in-patients of general medicine department with past medical history of stroke of a tertiary care teaching hospital in south India.

\section{MATERIALS AND METHODS Study design and setting}

A prospective observational study was performed in department of general medicine of a tertiary teaching healthcare setting in Anantapuramu, Andhra Pradesh, India

\section{Source of data}

A documentation from (data collection form) was designed to collect the patient's information which was kept confidential, the information from patients were collected only after explaining the merits and demerits of the study, and obtaining their consent for which an informed consent form was designed separately.

\section{Study duration}

Six months (January - June) 2016

\section{Ethical approval}

The ethical approval was obtained from the Institutional Review Board (IRB) of Raghavendra Institute of Pharmaceutical Education and Research (RIPER) before the commencement of the study (RIPER/ IRB/2016/08).

\section{Selection criteria}

Inpatient of general medicine department with previous stroke attack who are on medications, patients having stroke along with other clinical manifestations and risk factors causing repeated stroke attacks, patients newly diagnosed with stroke, with or without clinical manifestations of both genders and age greater than 18 years were included in the study.

\section{Sample size}

A total of 48 subjects satisfying the inclusion criteria's were included in the study.

\section{Study procedure}

The current prospective, observational, unicentric study of six months duration was performed among inpatients of general medicine department diagnosed with stroke of a tertiary teaching healthcare setting in south India, 
to assess their quality of life using Stroke specific QoL scale and Barthel index. A Structured process was followed for obtaining permission from hospital authority by submitting a detailed profoma of the study which includes protocol of study, evidence of critically evaluated biomedical literatures defining the need of study, data collection form, patient informed consent form. After the initial acceptance from the hospital, study was registered with the institutional review board (IRB) of RIPER, for ethical approval (RIPER/IRB/2016/08). Inpatients with stroke diagnosis of both genders greater than 18 years were enrolled, after explaining the merits and demerits of study, consent from patients or caregivers was obtained before their recruitment and documented. Patient information's regarding their demography, subjective and objective data's, past and present medical and medication histories; comorbidities and lifestyle were obtained and documented in data collection form. Study participants were administered with both Stroke specific QoL scale and Barthel index to assess their quality of life. The mean scores along with their standard deviations were calculated, ANOVA and student $T$ test was performed for each category and for different groups using GraphPad Instat.

\section{RESULTS}

The current prospective observational study of six months duration was performed to assess the quality of life in stroke inpatients of a tertiary care teaching hospital in south India.

\section{Demographic details of study participants}

Out of 48 stroke inpatients, the demography with respective to age and gender distribution illustrates $62.5 \%$ male and $37.5 \%$ female, furthermore there was equal distribution of patients with respect to gender within age groups of 56-60 and 61-65 years; results of which are thoroughly assessed and reported in Table 1. Demography distribution of study participants.

\section{Risk factors of stroke in study participants}

Risk factors of stroke could be categorized broadly in to modifiable and non-modifiable; Age, gender, race, ethnicity, and heredity have been identified as markers of risk for stroke. Although these factors cannot be modified, their presence helps identify those at greatest risk, enabling vigorous treatment of those risk factors that can be modified (Non-modifiable Risk Factors or Risk Markers). ${ }^{18}$

Stroke risks can be controlled more easily than one might think. Treating health conditions and managing unhealthy lifestyle risk factors can make a difference. ${ }^{19}$

In our study, the complications and risk associated with stroke is illustrated in Figure 1. Distribution of complications associated with stroke and Figure 2. Risk factors of stroke.

\section{Distribution of stroke}

In our study, 48 stroke inpatients were categorized based on stroke episodes and accidents, and previous attacks of stroke results of which are illustrated in Figure 3.

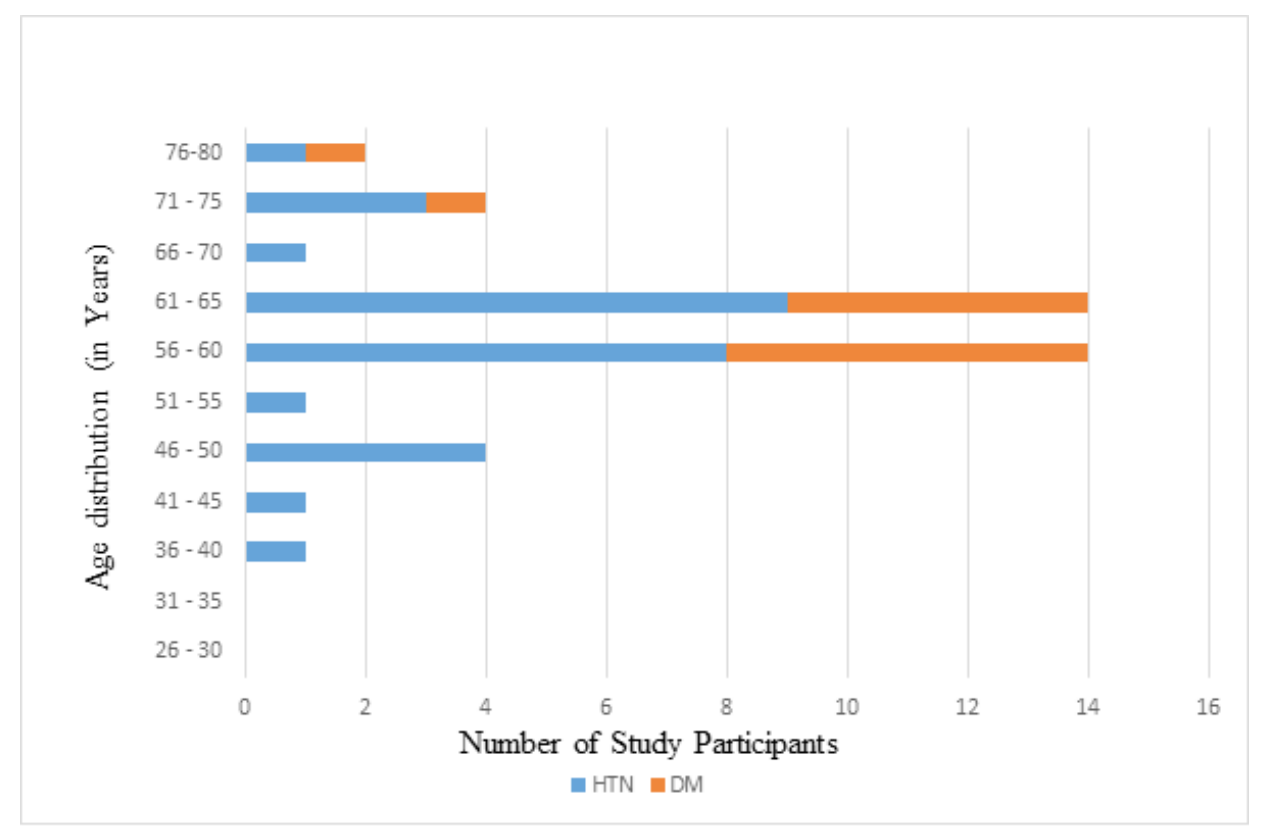

Figure 1: Distribution of complications associated with stroke 
Distribution of stroke episodes and accidents and Figure 4. stroke episodes.

Distribution of previous stroke attacks.

\section{Quality of Life (QoL) of stroke inpatients}

The Quality of Life in our 48 stroke inpatients were assessed with help of Stroke specific QoL scale and Barthel index, based on gender distribution, hypertension and diabetes mellitus complications, risk factors, and episodes of strokes; results of which are thoroughly assessed and reported in Table 2. Quality of Life (QoL) in stroke patients- Gender distribution, Table 3. Quality of Life (QoL) in stroke patients with complications, Table 4. Quality of Life (QoL) in stroke patients with risk factors and Table 5. Quality of Life (QoL) based on

\section{DISCUSSION}

To the best of our knowledge, this is the first research study aimed at assessing the quality of life in stroke inpatients of rural and healthcare resource limited settings of south India.

In our study, while assessing the QoL in stroke inpatients based on gender with both SSQOL and Barthel index, the mean score observed in SSQoL scale for females was less in comparison to males ( $\phi$ value $<0.0016$ ). Female patients mean score for Barthel index was almost similar

\begin{tabular}{ccc}
\hline $\begin{array}{c}\text { Table 1: Demography distribution of study participants. } \\
\begin{array}{c}\text { Age distribution } \\
\text { (in years) }\end{array}\end{array}$ & Total & \begin{tabular}{c} 
Gender distribution \\
\cline { 2 - 3 }
\end{tabular} \\
\hline $26-30$ & 3 & Male \\
$31-35$ & 2 & 1 \\
$36-40$ & 2 & 1 \\
$41-45$ & 1 & 0 \\
$46-50$ & 6 & 1 \\
$51-55$ & 2 & 5 \\
$56-60$ & 12 & 0 \\
$61-65$ & 12 & 9 \\
$66-70$ & 2 & 8 \\
$71-75$ & 4 & 2 \\
$76-80$ & 2 & 2 \\
Total & 48 & 1 \\
\hline
\end{tabular}

\begin{tabular}{ccccccc}
\hline \multicolumn{2}{c}{ Table 2: Quality of Life (QoL) in stroke patients- Gender distribution. } \\
$\begin{array}{c}\text { SSQoL for } \\
\text { males }\end{array}$ & $\boldsymbol{p}$ value & $\begin{array}{c}\text { SSQoL } \\
\text { for females }\end{array}$ & $\boldsymbol{p}$ value & $\begin{array}{c}\text { BI } \\
\text { for males }\end{array}$ & $\boldsymbol{p}$ value & $\begin{array}{c}\text { BI } \\
\text { for females }\end{array}$ \\
\hline $310.909 \pm 278$ & 0.004 & $156.72 \pm 121.64$ & 0.0016 & $16.09 \pm 16.15$ & 0.0081 & $9.2727 \pm 8.392$ \\
\hline
\end{tabular}

\begin{tabular}{|c|c|c|}
\hline Hypertension & & Mean + SD \\
\hline \multirow[t]{2}{*}{ Scales } & SSQoL & $191.1 \pm 159.71$ \\
\hline & $\mathrm{BI}$ & $8.818 \pm 7.22$ \\
\hline \multicolumn{2}{|l|}{ Diabetes mellitus } & Mean + SD \\
\hline \multirow[t]{2}{*}{ Scales } & SSQoL & $25.2727 \pm 44.150$ \\
\hline & $\mathrm{BI}$ & $1.54 \pm 2.659$ \\
\hline \multicolumn{2}{|c|}{ Hypertension and Diabetes mellitus } & Mean + SD \\
\hline \multirow[t]{2}{*}{ Scales } & SSQoL & $119.09 \pm 230.10$ \\
\hline & $\mathrm{BI}$ & $7 \pm 15.588$ \\
\hline \multicolumn{2}{|c|}{ No Hypertension and Diabetes mellitus } & Mean + SD \\
\hline \multirow[t]{2}{*}{ Scales } & SSQoL & $166.09 \pm 11$ \\
\hline & $\mathrm{BI}$ & $8.636 \pm 8.028$ \\
\hline
\end{tabular}




\begin{tabular}{|c|c|c|}
\hline \multicolumn{2}{|c|}{$1^{\text {st }}$ Episode stroke with accidents } & Mean + SD \\
\hline \multirow[t]{2}{*}{ Scales } & SSQoL & $109.45 \pm 100.86$ \\
\hline & $\mathrm{BI}$ & $7.4545 \pm 6.919$ \\
\hline \multicolumn{2}{|c|}{$1^{\text {st }}$ Episode stroke with no accidents } & Mean + SD \\
\hline \multirow[t]{2}{*}{ Scales } & SSQoL & $245.09 \pm 260.55$ \\
\hline & $\mathrm{BI}$ & $13.1818 \pm 16.672$ \\
\hline \multicolumn{2}{|c|}{$2^{\text {nd }}$ Episode stroke with accidents } & Mean + SD \\
\hline \multirow[t]{2}{*}{ Scales } & SSQoL & $47.2727 \pm 86.332$ \\
\hline & $\mathrm{BI}$ & $1.2727 \pm 2.687$ \\
\hline \multicolumn{2}{|c|}{$2^{\text {nd }}$ Episode stroke with no accidents } & Mean + SD \\
\hline \multirow[t]{2}{*}{ Scales } & SSQoL & $46.636 \pm 68.847$ \\
\hline & $\mathrm{BI}$ & $2.1818 \pm 3.970$ \\
\hline \multicolumn{2}{|l|}{ Multiple stroke episodes } & Mean + SD \\
\hline \multirow[t]{2}{*}{ Scales } & SSQoL & $35.7272 \pm 65.730$ \\
\hline & $\mathrm{BI}$ & $1.7272 \pm 3.849$ \\
\hline
\end{tabular}

\section{Table 4: Quality of Life (QoL) in stroke patients with}

\section{risk factors.}

\begin{tabular}{ccc} 
No risk & & Mean + SD \\
\hline Scales & SSQoL & $169.5454 \pm 128.35$ \\
& $\mathrm{BI}$ & $9.5454 \pm 8.092$ \\
One risk & & Mean + SD \\
Scales & SSQoL & $110.0909 \pm 116.68$ \\
& $\mathrm{BI}$ & $6.3636 \pm 7.915$ \\
Two risks & & Mean + SD \\
Scales & SSQoL & $141.3636 \pm 182.76$ \\
& $\mathrm{BI}$ & $7.8181 \pm 11.286$ \\
All risks & & Mean + SD \\
Scales & SSQoL & $63.1818 \pm 62.712$ \\
& $\mathrm{BI}$ & $2.6363 \pm 3.501$
\end{tabular}

but slightly less than that of males ( $p$ value $<0.0054)$.

The changes in quality of life observed in all stroke inpatients with respect to associated complications was found to be higher with hypertension by considering the 12 domains of SSQoL ( $p$ value $<0.0054$ ), which was less in observation among diabetic population. While comparing the population with both diabetic and hypertension and population having no hypertension and diabetes complications, the risk was found more in the later. The results of Barthel Index too was similar with SSQoL.

Different risk factors like smoking, alcoholism, pan chewing and tobacco chewing were considered in all patients to assess the severity of stroke attacks. The SSQoL mean for all the four differentiated risk factors were considered. Out of all stroke inpatients, the mean scores of patients with no risk are at more chance for stroke attack ( $\phi$ value $<0.0014)$, mean scores of patients with two risk factors are next to those with no risk ( $\phi$ value $<0.028$ ), followed by patients with one risk ( $p$ value $<$ 0.0107). The Barthel index means score values are similar to that of the SSQOL mean values.

The patients with accident as a risk factor was assessed for the cause of stroke attack, the mean scores for patients with $1^{\text {st }}$ episode without accident proved more risk for stroke attack in SSQoL scale ( $\phi$ value < 0.0109), the patients with $1^{\text {st }}$ episode with accident showed next more risk for stroke attack ( $\phi$ value $<0.0049)$, followed by patients with mean scores of $2^{\text {nd }}$ stroke episode with accident ( $\phi$ value $<0.0994)$, and patients with multiple episode showed no risk for stroke attack. The results of

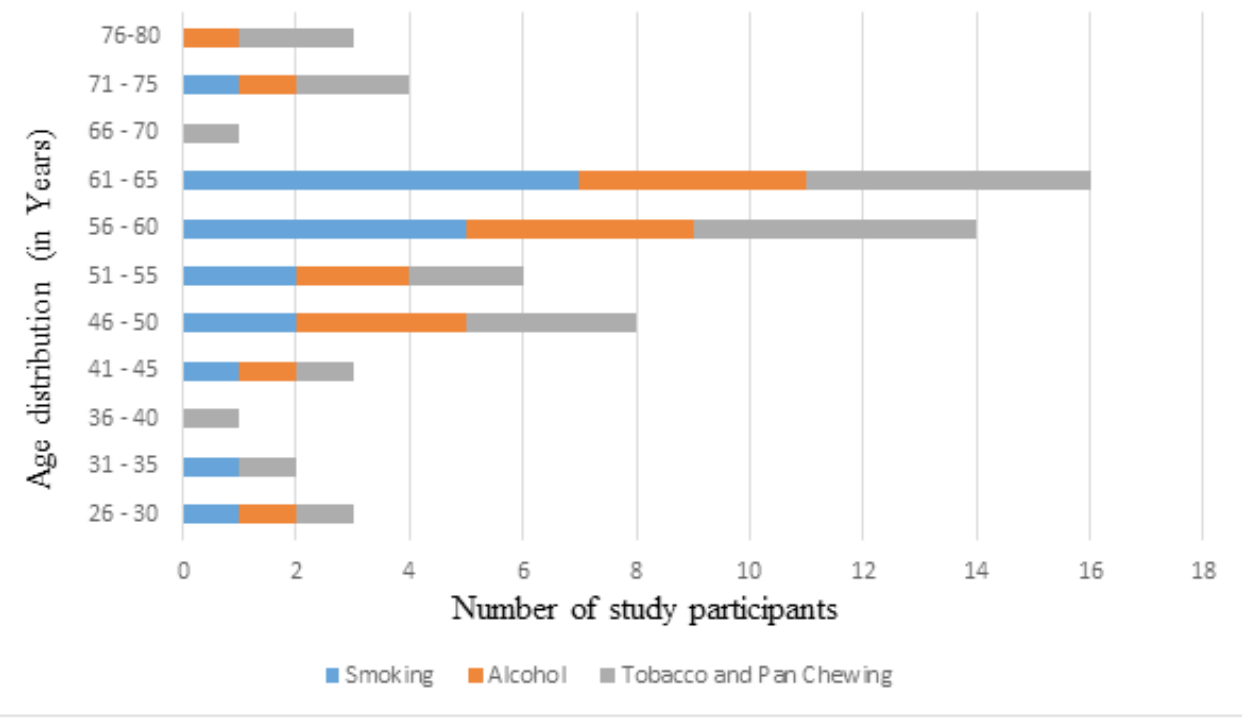

Figure 2: Risk factors of stroke 


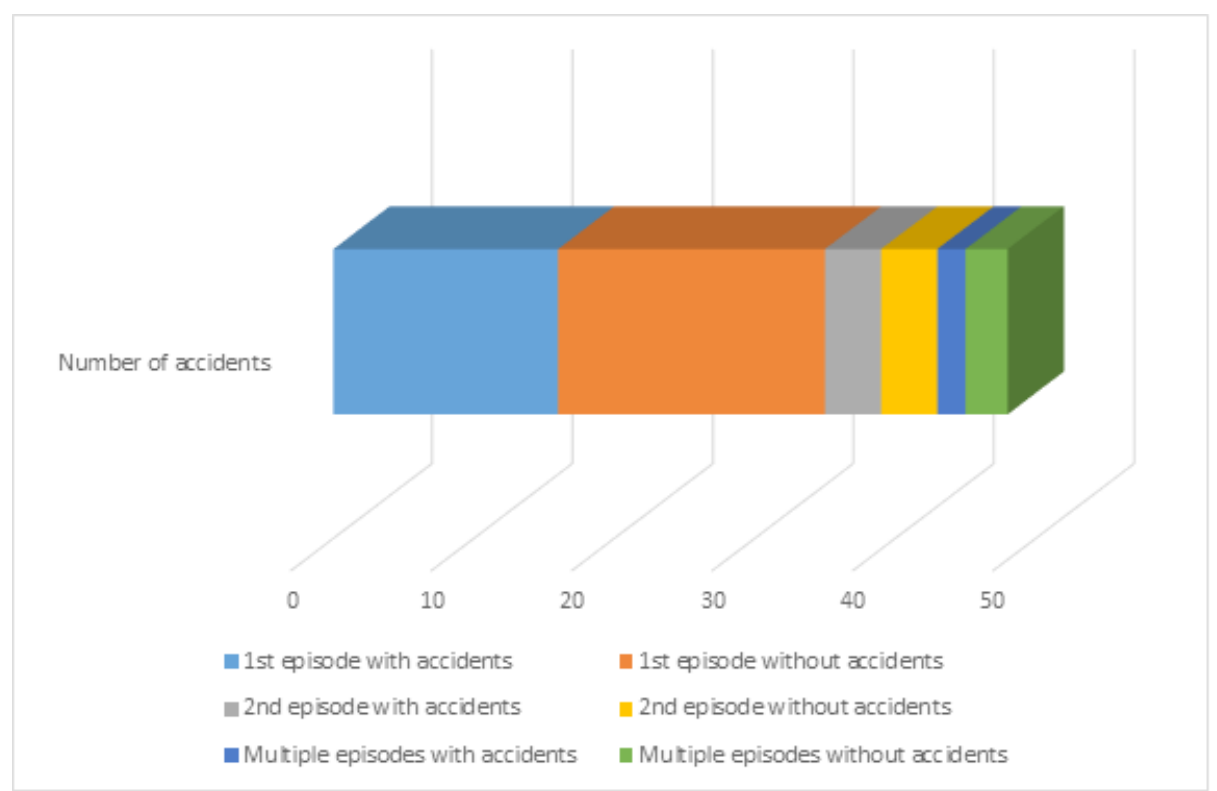

Figure 3: Distribution of stroke episodes and accidents

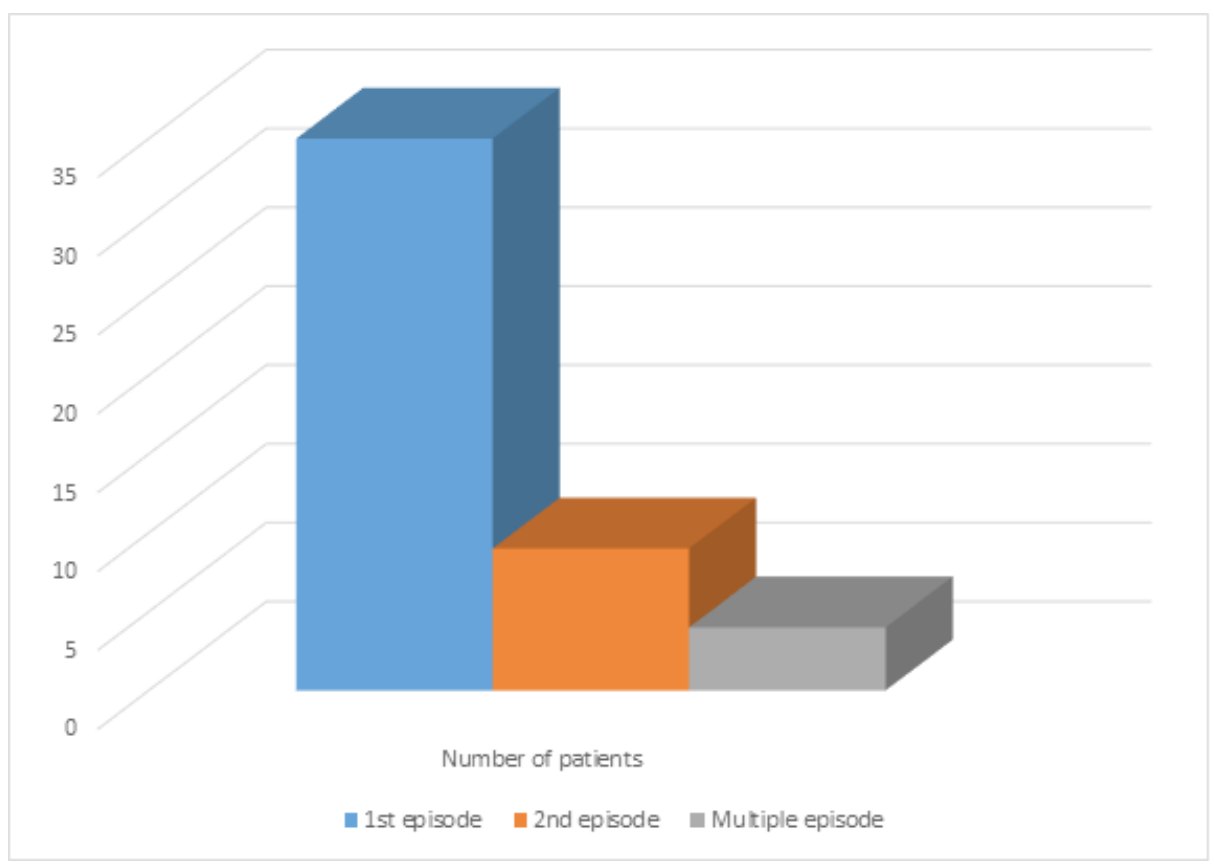

Figure 4: Distribution of previous stroke attacks

Barthel Index too was similar with SSQoL.

The primary outcome measures in this trial using SSQoL and Barthel index showed that the stroke unit had better function in dimensions that are accepted as important aspects of QoL. The SSQoL and Barthel index are often regarded as a measure of general perceived health status. The reliability and validity are quiet high just as high as on other scales of QoL with significantly fewer problems for the stroke unit group in the domains of energy, emotional reactions, social isolation, physical mobility and sleep the result of SSQoL and Barthel index. Scales strongly indicate a better QoL in that group than in general wards group because of the lack of understanding or failure in assessing the understanding. In our study nearly $20 \%$ of the patients could not be assessed for SSQoL. The Barthel index has often been used in assessment of stroke patients, although the Barthel index is not multidimensional factorial in comparison to other QoL scales, higher Barthel index scores is an indication of more active social life in that group.

In our study, a standardized systemic prescription and rehabilitation programme in stroke unit was carried at acute stages to improve patient's long term QoL. In our stroke unit we strongly emphasize psychological support and work closely with all patients to determine their abilities for health improvement and encourage them 
to return an active life despite their impairment and disability. With such an approach, we believe that our stroke unit enhances the psychological and social aspects that are important domains of QOL.

\section{CONCLUSION}

In conclusion, improved methods to measure the quality of life in stroke are required. Quality of life measures must be valid, reliable, responsive and comprehensive; and also with the involvement of patients at every stage of measure. Performing an exemplary and extensive research studies on stroke and its quality of life could bring better health outcome in individual patients.

\section{ACKNOWLEDGEMENT}

The authors would like to thank Dr. Harish, Assistant Professor and all medical staffs of General Medicine department of Govt Medical College and Hospital, Anantapuramu; all faculty members of department of pharmacy practice - RIPER and members of Institutional Review Board of Raghavendra Institute of Pharmaceutical Education and Research (RIPER) for their constant support and guidance.

\section{CONFLICT OF INTEREST}

The author declares no Conflict of Interest.

\section{FUNDS AND GRANTS}

Nil

\section{ABBREVIATIONS}

ANOVA: Analysis of Variance; BI: Barthel Index; CHD: Coronary Heart Disease; CT: Computed Axial Tomography; GBD: Global Burden of Disease; IRB: Institutional Review Board; QoL: Quality of Life; RIPER: Raghavendra Institute of Pharmaceutical Education and Research; SSQoL: Stroke Specific Quality of Life; WHO: World Health Organization.

\section{SUMMARY}

The quality of life after stroke and the methods of measuring this aspect of the disease have been viewed with a growing interest. Quality of life measures must be valid, reliable, responsive and comprehensive; and also with the involvement of patients at every stage of measure. Performing an exemplary and extensive research studies on stroke and its quality of life could bring better health outcome in individual patients.

\section{REFERENCES}

1. WHO MONICA Project Investigators. The World Health Organization MONICA Project (Monitoring trends and determinants in cardiovascular disease). J Clin Epidemiol. 1988;41(2):105-14.

2. Murray CJL, Lopez AD. The global burden of disease. 1996. Harvard school of public health.

3. http://www.sancd.org/Updated\%20Stroke $\% 20$ Fact $\% 20$ sheet $\% 202012$.pdf: stroke fact sheet India. Accessed 2013.

4. Li SC, Schoenberg BS, Wang C, et al. Cerebrovascular disease in the People's Republic of China: epidemiologic and clinical features. Neurology. 1985;35(12):1708-13.

5. Hu HH, Sheng WY, Chu FL, Lan CF, Chiang BN, Lo YK, et al. Incidence of stroke in Taiwan. Stroke. 1992;23(9):1237-41.

6. Suzuki K, Kutsuzawa T, Takita K, Ito M, Sakamoto T, Hirayama A, et al. Clinicoepidemiologic study of stroke in Akita, Japan. Stroke. 1987;18(2):402-6.

7. Kay R, Woo J, Kreel L, Wong HY, Teoh R, Nicholls MG. Stroke subtypes among Chinese living in Hong Kong: the Shatin Stroke Registry. Neurology JID 0401060. 1992;42(5):985-7.

8. Asian Acute Stroke Advisory Panel. Stroke epidemiological data of nine Asian countries. J Med Assoc Thai. 2000;83:1-7.

9. Shi FL, Hart RG, Sherman DG, Tegeler CH. Stroke in the People's Republic of China. Stroke. 1989;20(11):1581-85.

10. Lavados PM, Hennis AJM, Fernandes JG, et al. Stroke epidemiology, prevention, and management strategies at a regional level: Latin America and the Caribbean. Lancet Neurol. 2007;6(4):362-72.

11. Mar J, Sainz-Ezkerra M, Moler-Cuiral JA: Calculation of prevalence estimates through differential equations: application to stroke-related disability. Neuroepidemiology. 2008;31(1):57-66.

12. Muus I, Petzold M, Ringsberg KC: Health-related quality of life among Danish patients 3 and 12 months after TIA or mild stroke. Scand J Caring Sci. 2010;24(2):211-8.

13. Aprile I, Piazzini DB, Bertolini C, et al: Predictive variables on disability and quality of life in stroke outpatients undergoing rehabilitation. Neurol Sci. 2006;27(1):40-6.

14. Jaracz K, Kozubski W. Quality of life in stroke patients. Acta Neurol Scand. 2003; 107(5):324-9.

15. Ferrans CE. Definitions and conceptual models of quality of life. In: Lipscomb J, Gotay CC, Snyder C, editors. Outcomes assessment in cancer. Measures, methods, and applications. Cambridge: Cambridge University Press. 2005;14-29.

16. Kranciukaite $D$, Rastenyte $D$. Measurement of quality of life in stroke patients. Medicina. 2006;42(9):709-16.

17. Mahoney FI. Functional evaluation: the Barthel index. Maryland state medical journal. 1965;14:61-65.

18. Sacco RL, Benjamin EJ, Broderick JP, Dyken M, Easton JD, et al. Risk factors. Stroke. 1997;28(7):1507-17.

19. Stroke Risk Factors - National Stroke Association. http://support.stroke.org/ acute_site/risk-factors/ 